\section{SPECIFICITY OF POST-CONCUSSION SYMPTOMS AT 3 MONTHS AFTER MILD TRAUMATIC BRAIN INJURY. RESULTS FROM A PROSPECTIVE STUDY}

doi:10.1136/injuryprev-2012-040580g.21

\footnotetext{
${ }^{1,2}{ }^{2}$ L Lagarde* ${ }^{*}{ }^{1,2,3} \mathrm{~F}$ Masson, ${ }^{1,2,3}$ R Ribéreau-Gayon, ${ }^{1,2} \mathrm{D}$ Zongo, ${ }^{1,2,3}$ L -R Salmi, ${ }^{1,2} \mathrm{M}$ Laborey. ${ }^{1}$ University Bordeaux, ISPED, INSERM U897 Research Centre 'Epidemiology and Biostatistics', Bordeaux, France; ' ${ }^{2}$ INSERM, ISPED, Injury Prevention and Control Research Team, INSERM U897, Bordeaux, France, ${ }^{3}$ University Hospital Pellegrin, Bordeaux, France
}

Background Common complaints after mild traumatic brain injury (MTBI) include headaches, anxiety, irritability, and sleep disturbances, but these symptoms also occur in the non head-injured population. Whether they are more frequent among MTBI patients should help identify those that could be considered as part of the post concussion syndrome (PCS).

Aims/Objectives/Purpose We assessed the specificity of symptoms proposed in the three main scales used to measure PCS: post concussion symptoms listed in the Rivermead Post Concussion Symptoms Questionnaire (RPQ), the 4th edition of the Diagnostic and Statistical Manual of Mental Disorders (DSM-IV), and the 10th International Classification of Diseases (ICD-10).

Methods A cohort of 536 patients with MTBI and a comparison group of 946 patients with non-head injuries were recruited in the Adult Emergency Department (ED) of a teaching hospital in Bordeaux, France. Specificity of symptoms was assessed comparing MTBI and comparison patients at 3 months taking in to account baseline levels before ED admission.

Results/Outcomes 8 symptoms were identified as being specific to MTBI: headaches, dizziness, intolerance of stress, forgetfulness, poor concentration, taking longer to think, blurred vision, and personality change. Eight symptoms were found to be not specific to MTBI: nausea, noise sensitivity, irritability, anxiety, feeling frustrated, double vision, light sensitivity and restlessness.

Significance/Contribution to the Field The relevance of symptoms proposed to constitute PCS should be reviewed. A more specific definition of PCS would make diagnosis easier and facilitate prevention as well as treatment of patients with MTBI. 